\title{
Engine Management: A Decision Support Tool for Strategic Engine Maintenance Planning
}

\author{
Angel Figueroa Mayordomo ${ }^{1}$ and Dr. Adel A. Ghobbar ${ }^{2}$ \\ Delft University of Technology, Delft, 2629 HS, The Netherlands, \\ Ir. Stefaan S.A. Ghijs ${ }^{3}$ \\ Fly Aeolus, Antwerp, 2100, Belgium, \\ and \\ Eric Cator ${ }^{4}$ \\ Delft University of Technology, Delft, 2628 CD, The Netherlands
}

This paper presents a model that helps engine management make cost saving decisions. The model is developed around airline-influenced factors that have an impact on engine Maintenance Repair \& Overhaul (MRO): Operations, maintenance philosophy, contract type, and fleet age. Within the model a decision support tool is developed to analyze the impact of possible changes made to variables that influence engine MRO (engine thrust setting, shop visit schedule, and shop visit workscopes) on maintenance cost. The objective of the model is to support Engineering \& Maintenance in defining a maintenance philosophy, which includes engine time on-wing and life limited parts management strategies. This maintenance philosophy will help with maintenance schedule optimization aiming at matching engine maintenance status to phase-out conditions at end of lease contracts, while at the same time reducing engine direct maintenance cost.

$\begin{array}{ll}\text { AUR } & =\text { Aircraft Utilization Rate } \\ \mathrm{C}_{\text {contract }} & =\text { Contract Cost } \\ \mathrm{C}_{\text {fuel }} & =\text { Fuel Cost } \\ \mathrm{C}_{\mathrm{PhO}} & =\text { Phase-Out Cost } \\ \mathrm{C}_{\text {rent }} & =\text { Engine Rental Cost } \\ \mathrm{CPH} & =\text { Cost per Hour } \\ \mathrm{CSN} & =\text { Cycles Since New } \\ \mathrm{DMC} & =\text { Direct Maintenance Cost } \\ \mathrm{EGT} & =\text { Exhaust Gas Temperature } \\ \mathrm{E} \& M & =\text { Engineering \& Maintenance } \\ \mathrm{ESPH} & =\text { Engine Service Power by the Hour } \\ \mathrm{FC} & =\text { Flight Cycle } \\ \mathrm{FH} & =\text { Engine Flight Hour } \\ \mathrm{FHR} & =\text { Flight Hour Rate } \\ \mathrm{FCR} & =\text { Flight Cycle Rate } \\ \mathrm{H} / \mathrm{C} & =\text { Hour/Cycle Ration } \\ \text { LLP } & =\text { Life Limited Part }\end{array}$

\section{Nomenclature}

\begin{tabular}{|c|c|c|}
\hline HSN & $=$ & Hours Since New \\
\hline HSSV & $=$ & Hours Since last Shop Visit \\
\hline LLP $_{\text {invest }}$ & $=$ & LLP investment \\
\hline $\mathrm{LPP}_{\text {loss }}$ & $=$ & LLP cycle loss \\
\hline MRO & $=$ & Maintenance Repair \& Overhaul \\
\hline MTBSV & $=$ & Mean Time Between Shop Visits \\
\hline OEM & $=$ & Original Equipment Manufacturer \\
\hline $\mathrm{P}_{\mathrm{PhO}}$ & $=$ & Phase-out penalties \\
\hline $\mathrm{PP} \& \mathrm{C}$ & $=$ & Production Planning \& Control \\
\hline SV & $=$ & Shop Visit \\
\hline SVC & $=$ & Shop Visit Cost \\
\hline SVR & $=$ & Shop Visit Rate \\
\hline $\mathrm{R}_{\mathrm{T}}$ & $=$ & ESPH contract rate \\
\hline $\mathrm{R}_{\text {rent }}$ & $=$ & Engine rental rate \\
\hline TOW & $=$ & Time On-Wing \\
\hline TUSV & $=$ & Time Until Shop Visit \\
\hline Value $_{L P P}$ & $=$ & Value of LLP cycle \\
\hline
\end{tabular}

\footnotetext{
${ }^{1}$ MSc Student, Air Transport \& Operations, A.FigueroaMayordomo@student.tudelft.nl.

${ }^{2}$ Profile director Maintenance Repair \& Overhaul, Air Transport \& Operations, A.A.Ghobbar@tudelft.nl.

${ }^{3}$ General Director, Fly Aeolus, Antwerp Airport, Luchthavenlei 1, 2100, Antwerp, Belgium, ssaghijs@flyaeolus.com.

${ }^{4}$ Assistant Professor, Delft Institute of Applied Mathematics, Mekelweg 4, 2628 CD, Delft, E.A.Cator@tudelft.nl. 


\section{Introduction}

$\mathrm{E}^{\mathrm{n}}$ ngineering \& Maintenance (E\&M) plays an essential role in airline operations, as it must assure the availability of airworthy aircraft. Kinnison defines three core disciplines involved in conducting the technical maintenance activities at an airline: Maintenance, Engineering, and Management. ${ }^{9}$ They collaborate together, each carrying out their specific functions, with the aim of avoiding an aircraft on ground (AOG) situation. Maintenance takes care of the hands-on work. Engineering is responsible of supporting the maintenance work providing design, analytical, and technical assistance. Management is in charge of overseeing the maintenance operation providing the necessary organization, control, and administration. In his book, Kinnison identifies five main objectives associated with maintenance programs:

1. "To ensure the realization of the inherent safety and reliability levels of the equipment"

2. "To restore safety and reliability to their inherent levels when deterioration has occurred"

3. "To obtain the information necessary for adjustment and optimization of the maintenance program when these inherent levels are not met"

4. "To obtain the information necessary for design improvement of those items whose inherent reliability proves inadequate"

5. "To accomplish these objectives at a minimum total cost, including the costs of maintenance and the cost of residual failures"

There is one in particular which accurately defines the scope of this research: "To obtain the information necessary for adjustment and optimization of the maintenance program". The information is to help engineering and purchasing \& logistics departments in their Production Planning \& Control (PP\&C) task. PP\&C has three primary functions: forecasting, planning, and control. Forecasting focuses on estimating long and short-term maintenance needs for the existing fleet and business plans and any known changes in these for the forecasted period. Planning involves the maintenance scheduling and workscope definitions. Finally the control function allows adjustment of the plan in the case that events take place that require alterations to the plan. Both engineering and purchasing \& logistics cooperate together to obtain the information necessary to adjust $\mathrm{PP} \& \mathrm{C}$ to assure that maintenance activities are carried out with the lowest possible cost and highest productivity, aiming to contribute to maximizing the profit of an airline.

For a Low Cost Low Fare carrier, a young, modern fleet is essential for low-cost operations, as it helps minimize downtime and operational expenses. To obtain and maintain such a fleet an effective fleet renewal program has to be put into place. Correct maintenance scheduling decisions become an important factor, since at aircraft phase-out maintenance issues arise regarding the aircraft's engines status. Aircraft engines must be accounted for and reported on in much the same fashion as aircraft. Whether an aircraft is owned or leased the engines must meet certain phaseout conditions. In this case E\&M must make sure that the engines mounted on the aircraft meet these conditions, which include specific limits such as remaining time on-wing (TOW) or life of Life Limited Parts (LLP).

One of the main problems encountered in engine maintenance scheduling is deciding when to replace the engine's LLPs. An LLP is an engine component that has a predefined maximum operational life, its hard life. The component must be replaced once the maximum number of flight cycles is reached. In practice these parts are replaced with a certain amount of economically unusable life remaining, its stub life. Reducing this stub life combined with the need to meet phase-out conditions, raises the issue of whether replacing LLPs at their hard life is optimal from an economical perspective. Airlines facing end of aircraft lease contracts, must keep return conditions in mind, and decide on the optimum moment to invest in LLPs.

An engine rarely remains installed on the same aircraft throughout the aircraft's service life. The flexibility provided by the possibility of swapping around engines and LLPs, is a factor which allows airlines to manage cost. This flexibility is important in order to avoid unnecessary costs at the end of operational lease periods, as would be the case if the engine phase-out requirements were not met. Deciding which engine to mount on which aircraft and defining the workscopes of the shop visits (SV) allows for some flexibility in managing cost. Correctly forecasting LLP needs and scheduling the maintenance accordingly could reduce the number of necessary SVs, and avoid unnecessary investment in LLPs. Trying to avoid these additional costs creates an interesting scheduling and cost reduction challenge.

The objective of this paper is to provide a model that supports airline Engineering \& Maintenance departments in forecasting, planning, and controlling engine maintenance, with the aim of reducing engine direct maintenance cost. This model can provide an improved view of engine maintenance status, support maintenance scheduling tasks and support engine management in making accurate, cost saving decisions. This objective is achieved in cooperation with an airline that provided the necessary data to analyze in order to determine: What are the drivers behind engine availability and direct maintenance cost? What are the maintenance and contractual limits faced in engine 
maintenance? Is there flexibility within engine maintenance scheduling? If yes, can it be used to reduce cost? And, are the equations used to forecast engine status and maintenance cost correct?

The model that is presented in this paper is calibrated and validated using actual engine operations data, from 59 engines, supplied by an airline. This data is first used to make sure that the programmed tool accurately calculates engine maintenance status using the actual monthly engine utilization. The program will then be validated taking a group of six engines, all belonging to the same lease contract, and measuring the error in engine status and maintenance cost forecasting throughout a period of two years.

The remainder of the paper is outlined as follows: Section 2 provides a quick look at some of the more relevant engine management theory on which the model is based. Section 3 introduces the maintenance-planning model. And section 4 provides a more in depth look at the decision support tool developed to support engine maintenance planning.

\section{Engine Management}

Before commencing with the development of the model a literature review was carried out to obtain a better understanding of the CFM56-7B engine and the factors that influence its availability and maintenance cost. ${ }^{1,2,4-6,8,9,11}$ This engine was chosen due to the fact that the fleet used to calibrate and validate the maintenance planning tool operates on this engine type. The most relevant theory from the literature review needed to grasp the concept of the model is presented in this section.

\section{A. Maintenance Processes}

There are three recognized processes used in the airline industry to define maintenance: Hard Time, On Condition, and Condition Monitoring. The modern trend is to maintain engines on a Condition Monitoring basis, which means that engines are maintained based on failure-based monitoring and predictive monitoring. Failure based monitoring is when components are allowed to remain in service until a functional failure. Predictive monitoring consists of monitoring deterioration of operating parameters such as $\mathrm{N}_{1}$ and $\mathrm{N}_{2}$ speeds or Exhaust Gas Temperature (EGT) to identify incipient failures in order to schedule economical repairs before the occurrence of extensive costly damage. The LLPs are the exception to condition monitoring maintenance. These parts require Hard Time maintenance, meaning they must be replaced before they exceed their life cycle limit defined by the original equipment manufacturer (OEM).

\section{B. Engine Shop Visit Forecasting}

Forecasting is the art of predicting what the future demand will be. Future demand is often difficult to predict, especially when it continuously fluctuates or it is affected by many external factors. An important part of any forecasting process is establishing the objectives of the forecast. For engine maintenance the forecast has two main purposes: 1) predict future engine maintenance status, and 2) predict the SVs that must be scheduled.

The capability of accurately forecasting upcoming SVs is a valuable aspect of maintenance planning. Forecasting can be done using Engine Utilization, which is a measurement of the length of time an engine is operated. This can be quantified in both flight hours $(\mathrm{FH})$ and flight cycles (FC). The FH represents one hour of flight, whereas FC represents one aircraft take-off and subsequent landing. The Engine Utilization is defined in an aircraft's operational profile, which can be described as an hour-tocycle ratio $(\mathrm{H} / \mathrm{C})$. Figure 1 illustrates the comparison between two flight profiles with different $\mathrm{H} / \mathrm{C}$ 's.

The FH, FC, and $\mathrm{H} / \mathrm{C}$ are generally used to estimate when an engine must go to shop. FHs are the unit of measurement used to predict when SVs resulting from condition monitoring will occur. FCs are used to indicate SVs resulting from Hard Time based maintenance of components with life cycle limits, in the case of an engine

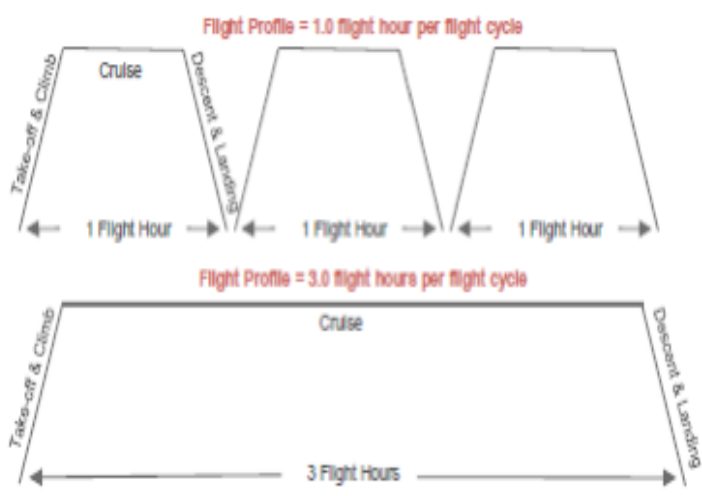

Figure 1. Two flights profiles with different operating profiles

its LLPs. The $\mathrm{H} / \mathrm{C}$ allows for only one of the measurements to be required. Using the $\mathrm{H} / \mathrm{C}$ one can figure out the $\mathrm{FH}$ from the FC or the FC from the FH. Therefore from an aircraft's operational profile one can forecast the total $\mathrm{FH}$ and FC an aircraft will operate on a monthly or yearly basis. 


\section{Direct Maintenance Costs}

Engine Direct Maintenance Cost (DMC) is the cost for scheduled and unscheduled labor, material and outside services applied to meet the performance of maintenance directly related to the operation of an engine. Generally this cost is the measure of an airline's maintenance philosophy.

The maintenance philosophy consists of an airline's strategy regarding its engine SV planning, deciding what is the optimum balance between $\mathrm{SV}$ cost (SVC) and SV rate (SVR). The philosophy is determined by taking into account both operational and financial considerations. The operational considerations focus on obtaining an efficient operational performance out of the engines, measured by the number of AOGs caused by engine failures, whereas the financial considerations focus on reducing the DMC. In the end the maintenance philosophy followed is key to lowering maintenance cost.

The measure of a maintenance philosophy is the Cost Per Hour (CPH), and its main drivers are: the operational variables, the SVC and the SVR. The

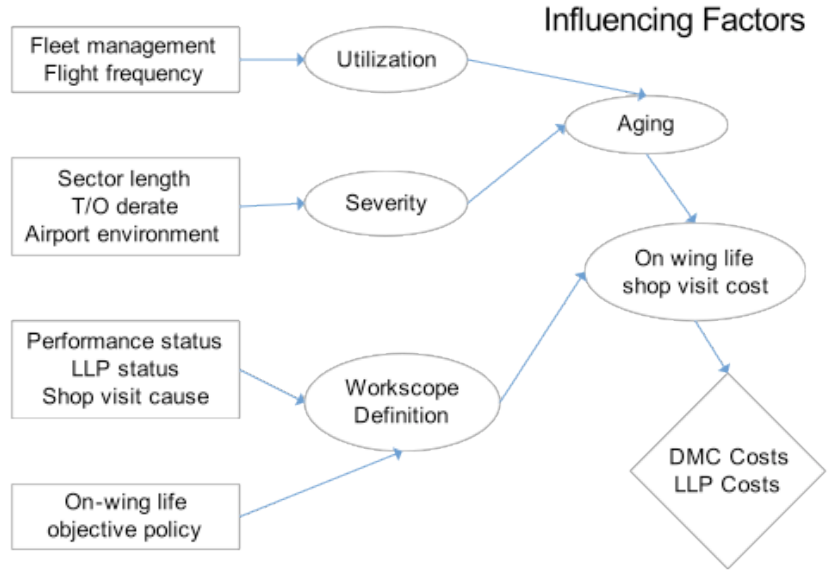

Figure 2. Cost drivers influencing factors operational variables include: annual utilization, flight leg, modeled thrust, take-off de-rate, and environmental conditions. The SVR is determined by the Time On-Wing (TOW) policy and the LLP expectations. Whereas the SVC depends on the engine performance and component status, since these determine the required level of engine restoration and therefore the corresponding SV workscope. All these are key factors in the final engine DMC and LLP costs (figure 2).

\section{Shop Visit Workscoping}

Once an engine is sent to shop the level of workscope to be performed is dependent on the removal cause(s); time accumulated on wing of the different engine modules, observed hardware conditions, engine performance, and business goals. One of the main objectives of this model is to analyze the possibility of reducing engine DMC costs by optimizing the use of its LLPs. As an example figures 3 and 4 show two different scenarios of LLP management: Scenario A) Optimized for maximum usage of LLPs, and B) Optimized for minimum number of SVs.

Each engine is divided into four modules in the following order: 1) Fan/Low Pressure Compressor (LPC), 2) High Pressure Compressor (HPC), 3) High Pressure Turbine (HPT), and 4) Low Pressure Turbine (LPT). Each module has a different hard time maintenance value, defined by the module's LLPs. The value of life limiting cycles can be seen in the figures as the numbers written inside their respective modules (x1000). Each new engine in the figure shows an SV moment. The values in-between show the flight cycles the engine operates in between each SV. The grey colored in modules represent the modules in which LLPs are replaced (RPL), with the core LLPs referring to the LLPs in the HPC and HPT modules.

It is important to notice that while scenario A tries to maximize its LLP use to reduce investments on LLPs, scenario $\mathrm{B}$ ends up having the lower total DMC cost by minimizing the number of SV. The lower cost is due to the fact that even though scenario B invests more money on LLPs, it makes up for it by requiring one less $\mathrm{SV}$.

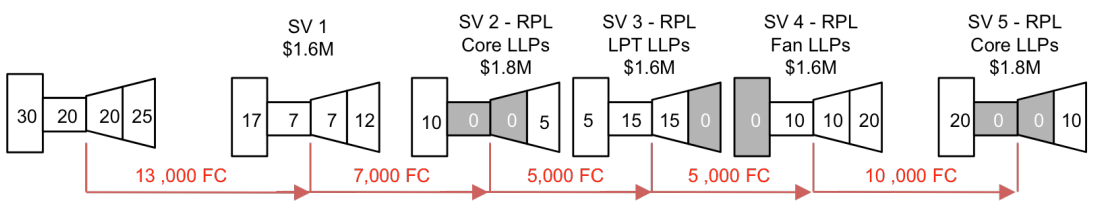

Figure 3. Scenario A: Optimized for maximum usage of LLP This results in the lower total

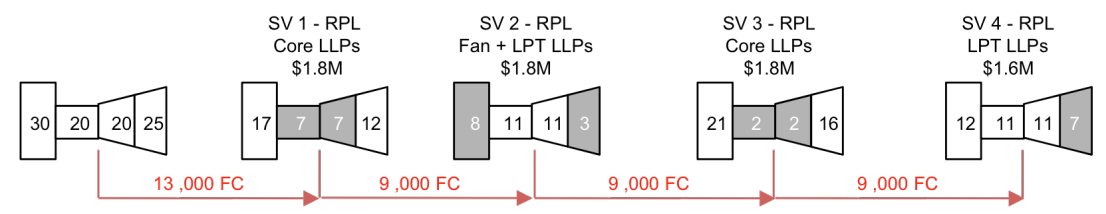

Figure 4. Scenario B: Optimized for minimum number of shop visits 
DMC cost, table 1. This shows that it is not necessarily true that running the LLPs to their limits will reduce cost. The real cost reduction is finding the balance between LLP usage and number of SVs. One of the key factors that make this model useful is that it manages to obtain an efficient manner of comparing the different LLP usage possibilities, making easier to compare them and therefore define a cost-efficient maintenance plan.

\begin{tabular}{|lllllll|} 
& FC & FH & Restoration & LLPs & Total SV & Total DMC \\
\hline Scenario A & 40,000 & 80,000 & $8,400,000 \$$ & $3,000,000 \$$ & $11,400,000 \$$ & $142.50 \$ /$ FH \\
Scenario B & 40,000 & 80,000 & $7,000,000 \$$ & $3,800,000 \$$ & $10,800,000 \$$ & $135.00 \$ / F H$ \\
\hline
\end{tabular}

Table 1. Total DMC for different workscoping scenarios

\section{Maintenance Planning Model}

Now that the basic theory behind engine maintenance processes, forecasting, DMC, and workscoping has been laid down this section will go on to describe the engine maintenance-planning model. The idea is to model engine planning in a similar way to an airline-planning model put forward by Boeing. ${ }^{3}$ The structure of this planning model, with some slight modifications, makes a useful layout for the development of an engine maintenance-planning model. The overall structure of the model is depicted in figure 6 . The first step in planning is to identify what must be accomplished. In this case it was decided that the goal is to create a strategic engine maintenance plan. To achieve this the model is split up into three phases: 1) Network Forecast, 2) Fleet Analysis, and 3) Maintenance Planning.

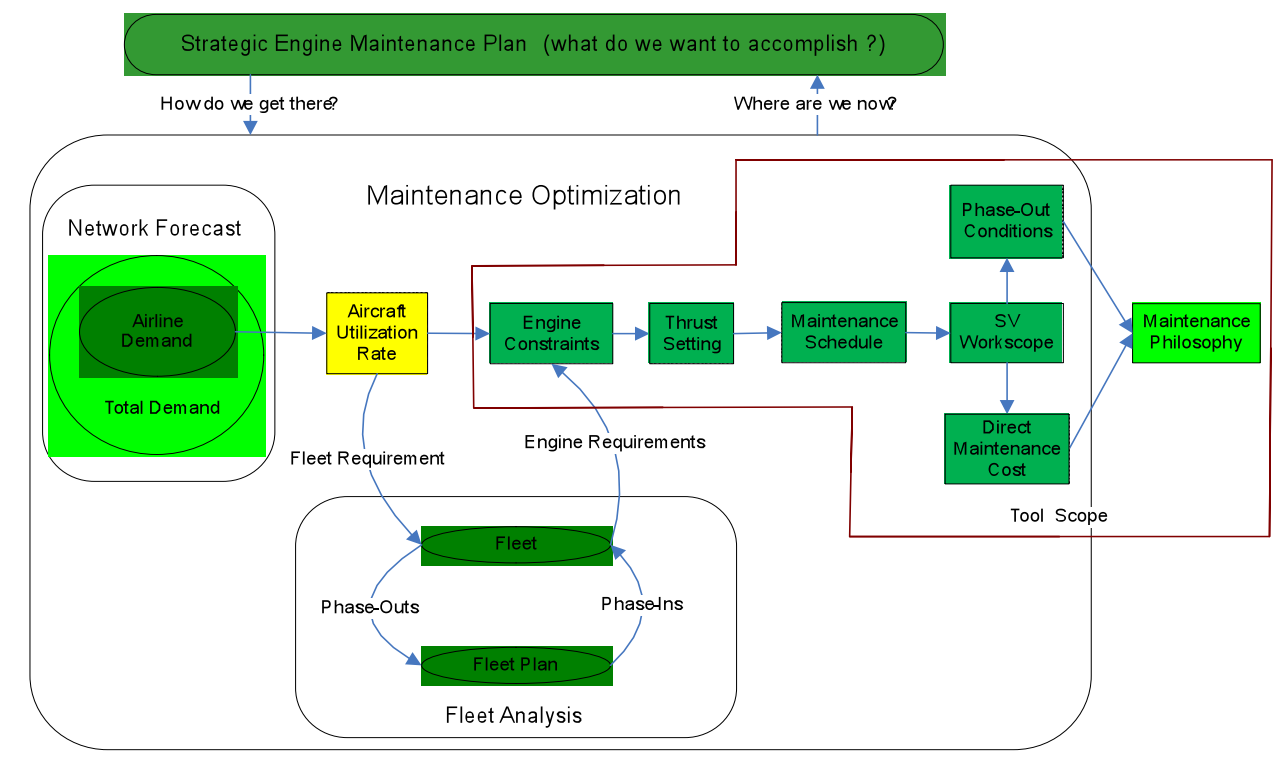

Figure 5. Engine Maintenance Planning Model

\section{A. Influencing factors in engine MRO}

The key players that influence engine maintenance repair and overhaul (MRO) are: Airline, lessor, MRO provider, original equipment manufacturer (OEM), and regulator. ${ }^{10}$ This model is developed to manage the airline influenced factors that affect engine MRO: Operations, maintenance philosophy, contract type, and fleet age. ${ }^{10}$ All these factors are accounted for in either the Network Forecast, Fleet Analysis or Maintenance Planning phases of the model. In addition, the Maintenance Planning phase also takes into account the

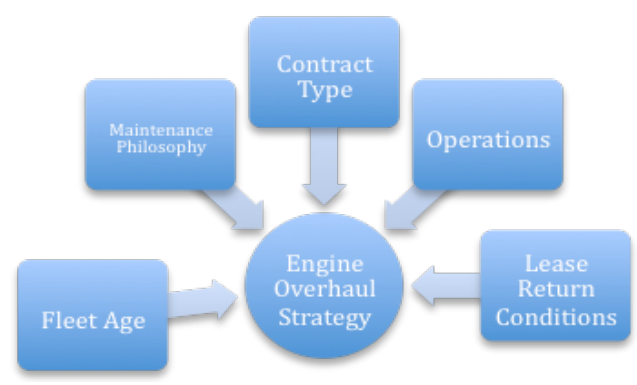

Figure 6. Airline influenced factors in engine MRO 
lease return conditions imposed by the lessor. These define a limit on engine maintenance status at the end of the lease period, hence forcing engine overhaul scheduling to take into account that the lease return conditions imposed by the lessor must be met.

\section{B. Network Forecast}

In the Network Forecast phase the focus is placed on analyzing market demand trends. The goal of the analysis is to forecast an airline's flight demand, its operations, as accurately as possible. This flight demand can then be translated into an Aircraft Utilization Rate (AUR). The forecasted AUR defines the operations of an airline, giving the number of flight hours and flight cycles an engine will operate in a given period of time. For this model this will be an external input based on the airline's demand forecast.

\section{Fleet Analysis}

In the Fleet Analysis phase the fleet is shaped so that it can meet the forecasted flight demand. Within this analysis a fleet strategy is drawn-up which defines the fleet age, maintenance contract type, and lease return conditions. As far as engine management is concerned the main output of the fleet analysis are the definition of the engine 'fleet' size, engine type, contract rates, and phase-out requirements. These, like the Network Forecast, will also be an external input provided by the fleet manager.

\section{Maintenance Planning}

The final phase, Maintenance Planning, uses a tool to define a maintenance philosophy. The general structure of this tool can be broken-down into three steps: Data initialization, maintenance definition, and phase-out definition (figure 7). With the final output being the cost per hour $(\mathrm{CPH})$ of the final maintenance plan. This phase is defined based on the engine maintenance scheduling procedures at an airline, and then analyzing the deficiencies experienced in practice. With the results of the analysis the different steps required to obtain an efficient maintenance plan are defined.

The data initialization consists of inserting the inputs defined in the Network Forecast and Fleet Analysis phases into the tool. These

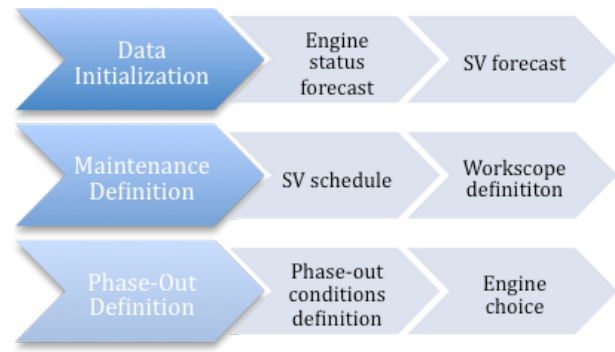

Figure 7. Decision support tool workflow inputs, combined with the engine data (initial conditions, operational constraints and thrust setting), are used to forecast engine status, which is then used to forecast engine SVs.

The maintenance definition uses the forecasted engine SVs to define a maintenance plan. It consists of three steps: 1) define engine thrust setting, 2) decide on an SV schedule, and 3) define the workscopes for the planned SVs. Combining these three steps updates the engine status forecast.

The phase-out definition uses the updated engine status forecast to predict engine status at the scheduled engine phase-outs. At this point the phase-out conditions are defined and an overview is made of an engine's status in relation to these conditions. From this overview a choice is made on which engine to phase-out at which moment. Then a cost calculation is carried out for each specific engine based on the maintenance cost of the planned SVs and any additional costs faced at phase-out. The final output is the cost for the proposed maintenance plan for the entire engine fleet.

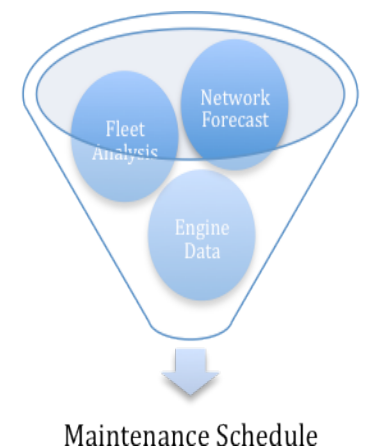

Figure 8. Inputs and Outputs
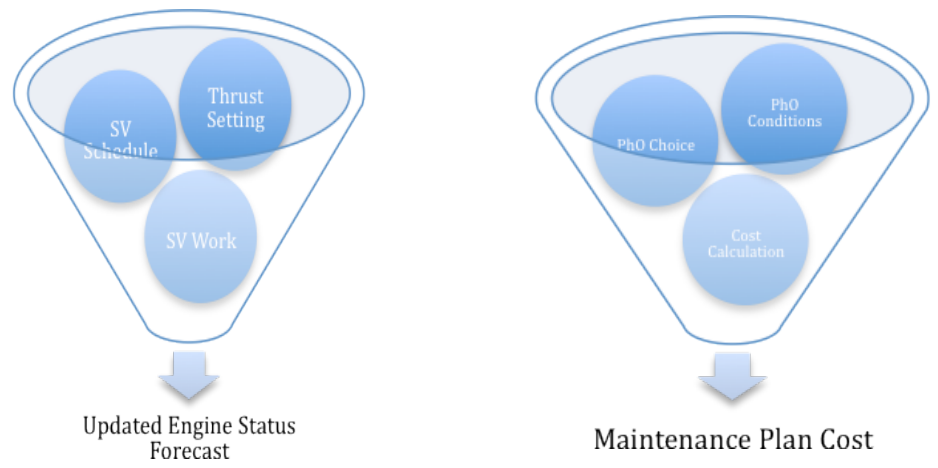

Maintenance Plan Cost 
Figure 8 illustrates the three model stages and their corresponding outputs. A more detailed description of the tool, its workflow and the variables it takes into account, is provided in the following section, Decision Support Tool. The impact of the decisions made can be analyzed and revised in order to come up with an improved operational performance or cost. The final output of this process should define an engine maintenance philosophy, which is what will shape the airline's final strategic engine maintenance plan.

\section{Decision Support Tool}

The previous section introduced the maintenance-planning model. This section will define the tool that has been developed to support the Maintenance Planning phase of the model. The tool is a Matlab program used to forecast, plan, and control engine maintenance. It will help an airline evaluate its maintenance philosophy and study the impact of possible changes to the maintenance cost. The workflow of this tool can be divided into eight stages: Data analysis, data initialization, forecast, maintenance definition, phase-out definition, cost calculation, scenario generation, and control. These stages were defined based on the analysis of an engine management process in practice, and consultation with specialists in engine PP\&C.

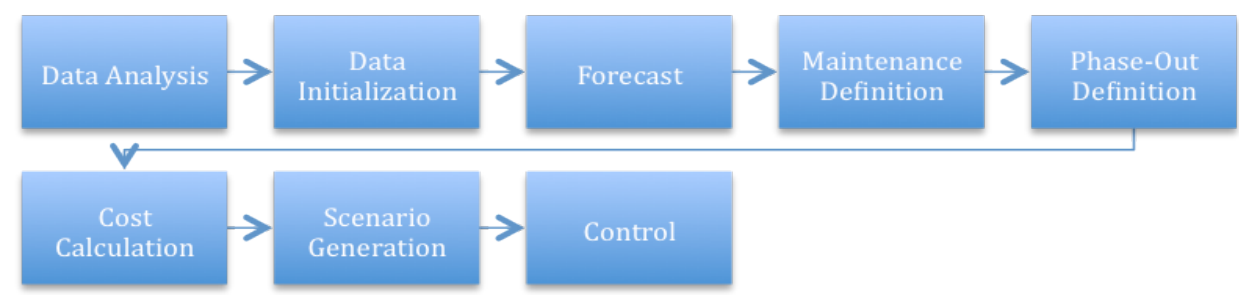

Figure 9. Decision support tool workflow

\section{A. Data Analysis}

The data initialization is preceded by a data analysis of the engine fleet in question. The data that must be analyzed can be divided into three fields:

- Operations: Engine yearly utilization (Cycles and hours)

- Maintenance: Engine SV (Workscopes, forecast, and cost)

- Contracts: Maintenance and lease contracts

The data is used to answer the following questions: What are the drivers behind engine availability and direct maintenance cost? And, what are the maintenance and contractual limits faced in engine maintenance?

The operations data is analyzed in order to define engine utilization, which is one of the inputs of the data initialization stage. This input is the base of the program since the whole forecast of engine maintenance status is based on how many cycles and hours an engine operates each year. The engine SV data consists of the workscopes of past engine SVs along with their respective costs, and current predictions of future SVs. This data will be used to define engine maintenance limits, the main

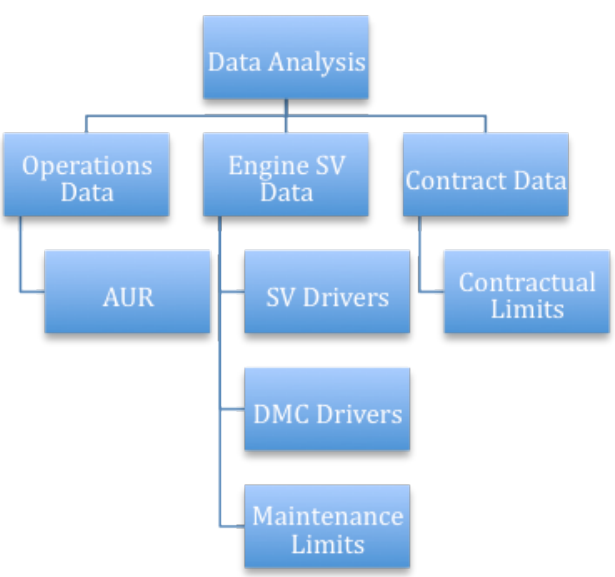

Figure 10. Data analysis SV drivers that should be forecasted, and the DMC drivers. The contractual data is based on the current engine lease contracts, which are used to determine which are the maintenance status limits that must be met at the end of a lease contract. These drivers and limits define the flexibility of the decision support tool. The drivers are used to identify what variables must be programmed into the tool and the limits define the fixed values that define when an engine must go to shop or exceeds it lease contract return conditions. It must be kept in mind that these values will change depending on the airline, since each airline flies different routes, has different engine types, and different maintenance contracts. 


\section{B. Data Initialization}

Once all the variables have been identified in the data analysis phase the next step is the data initialization stage. This stage consists of introducing the variables identified in the data analysis phase into the program. All the inputs are gathered from the three key players involved: Management, Engineering, and Purchasing. The following section defines the data initialization variables identified from a data analysis of an actual airline.

The Management inputs are all the variables that belong to the Forecast and Fleet Analysis phases of the model. These inputs are, in theory, fixed values. The inputs are: Number of engines, number of phase-outs (PhO), aircraft utilization rate (AUR), hour/cycle (H/C) ratio, and phase-out conditions. The number of engines defines for how many engines the tool is going to be forecasting. The number of

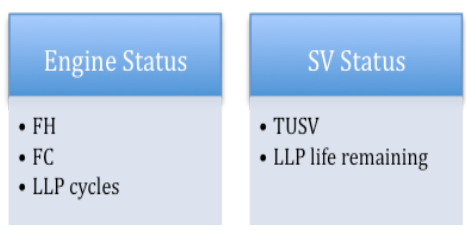

Figure 12. Status databases PhOs defines how many engines are going to be retired from the fleet in the time span of the forecast. The AUR defines the number of cycles an engine will operate on a monthly basis. The $\mathrm{H} / \mathrm{C}$ is used to convert from the number of cycles to the number hours of hours an engine operates. And the phase-out conditions define the maintenance condition that the chosen engines must satisfy at phased-out.

The engineering department inputs define an engine's status and operating limits when the program is first started, they are: Engine initial conditions, performance limits, and thrust settings. The engine initial conditions are the starting point of the relevant factors needed for the engine status and SV forecasts: Hour since new (HSN) cycles since new (CSN), LLP status (remaining cycles), and hours since last SV (HSSV). The performance limits are based either on FH or FC and are used to predict when the next SV should take place. In the case that the engine removal reasons are based on FHs, the prediction uses the Mean Time Between Shop Visit (MTBSV). An example of such an SV limit is the MTBSV for the J-hook replacement. If the engine removal reasons are based on FCs then the prediction uses the CSN and life cycle limit of the specific component. An example of such an SV is the replacement of LLPs. The equations used to forecast the different types of SVs are explained in more detail in the following sub-section, Forecast. The thrust setting refers to the thrust rating on which each engine operates.

The purchasing inputs are those that are handled by the purchasing managers and will be used to calculate the engines' DMC. Normally these inputs would be along the lines of material, labor, and component prices, however the fact that this tool is modeled for airlines whose engine maintenance is covered by an engine service power by the hour (ESPH) contract reduces the complexity of the cost modelling. These contracts allow airlines to pay for maintenance based on engine FHs instead of on a time \& material basis. The only components that might not be covered by the contract are the LLPs. Therefore the inputs are: Initial 'bucket', contract rates, LLP values, and engine rental cost. The initial bucket is the maintenance reserve an engine has build up based on the monthly contract rates and FH flown. The engine rental cost is the cost of leasing in an engine if the number of engines at shop exceeds the number of spare engines at the airlines disposal. The cost calculation equations for which these inputs are used are explained in more detail in the Cost Calculation sub-section.

The tool is used to bring all these variables together and use them to forecast an engine's status and the resulting DMC based on its planned operations and maintenance workscopes. The following sub-sections will give an overview of the equations used to forecast engine status, SV moments, and cost.

\section{Forecast}

Once all the inputs have been introduced into the program the engine status, SV status, and financial status databases are generated. These databases keep track of: Flight Hours (FH), Flight Cycles (FC), LLP cycles, time until SV (TUSV), LLP life remaining, contract cost, and the bucket. The equations presented in this section are defined in the program and used to predict the different factors in future years. They are based on
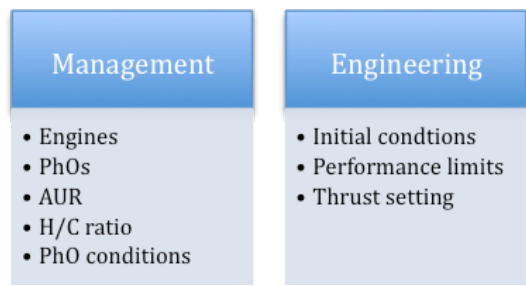

Figure 11. Tool input variables consultation with experts in the engine maintenance field.

In order to predict an engine's SV moments and DMC the first step is to predict that engine's cycle AUR. With the AUR one can forecast the CSN by simply adding the predicted number of cycles an engine will operate to its initial CSN, Eq. (1). The HSN can also be forecasted from the AUR by multiplying it by the H/C ratio, Eq. (2). Once these values are accurately predicted it is possible to try and forecast SV moments and DMC. 


$$
\begin{gathered}
C S N=C S N_{O}+A U R \\
H S N=H S N_{O}+A U R \times H / C
\end{gathered}
$$

This tool is designed to forecast SV moments for Hard Time and condition monitoring (failure-based and predictive) maintenance processes. Hard Time maintenance is required for the LLPs, these consist of rotating (disks, seals, spools, and shafts) and static (shrouds and frames) parts, whose failure could create a hazard to the aircraft. Therefore the parts must be replaced before they exceed their life cycle limits. These limits change slightly depending on the thrust rating the engine is operating on. Eq. (3) shows how the remaining cycles are calculated, the cycles that an engine has operated on a thrust rating are divided by their respective cycle limits. The limit is $_{\mathrm{T}}$ the life cycle limit for the thrust rating for which the cycles remaining are being calculated. $\mathrm{Cycles}_{24 \mathrm{k}}$, Cycles $26 \mathrm{k}$, and Cycles $_{27 \mathrm{k}}$ stand for the cycles the LLP has been in operation on the different thrust ratings (24k, 26k, and 27k). Eq. (4) is used to forecast SVs based on predictive monitoring, the deterioration data is used to determine the MTBSV for the different engine removal reasons. Using the MTBSV and the HSSV a prediction is made of when the next SV should occur. The failure-based SVs are dealt with in the control stage of the tool workflow.

$$
\begin{gathered}
L L P_{\text {cycles }}=\left(1-\frac{\text { Cycles }_{24 k}}{\text { Limit }_{24 k}}-\frac{\text { Cycles }_{26 k}}{\text { Limit }_{26 k}}-\frac{\text { Cycles }_{27 k}}{\text { Limit }_{27 k}}\right) \times \text { Limit }_{T} \\
T U S V=M T B S V-H S S V
\end{gathered}
$$

The financial status database is used later on to predict the resulting cost of the maintenance plan. The equations used to calculate contract cost are introduced in the Cost Calculation sub-section.

\section{Maintenance Definition}

As stated in the Maintenance Planning Model section the maintenance definition consists of two steps: 1) decide on SV schedule, and 2) define the workscopes for those SVs. For this the SV forecast is used to predict the moments that SVs will be needed. The SVs with their respective reasons will be displayed in a table that shows for which engine and in what month an SV is due. At this point the SV schedule is made and the workscopes defined. Based on these decisions the tool will update all the status databases and create a new engine maintenance status forecast.
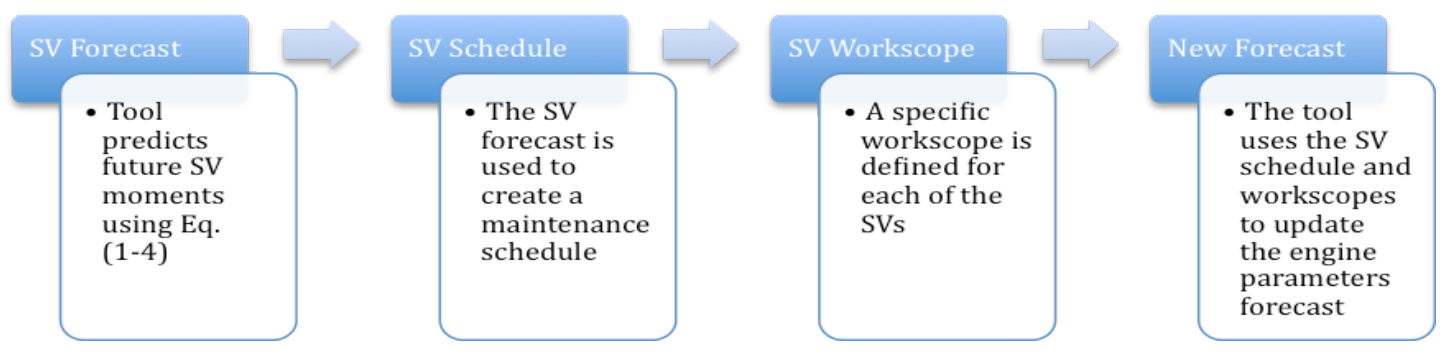

Figure 13. Maintenance definition stage

\section{E. Phase-Out Definition}

In the phase-out definition stage the phase-out dates are defined along with the corresponding phase-out conditions. The program then displays, in a graph, the month each engine meets the phase-out conditions in relation to the actual phase-out dates.

The phase-out conditions used to generate the graph are dependent on the engine's lease contract. These conditions usually include limits regarding the engine's maintenance status, such as: Time since overhaul (TSO), LLPs remaining life, or EGT margin. These limits are defined and used to calculate until what moment each of the engines satisfies them. An example of a possible phase-out condition is the percentage of life remaining on the LLPs. This is calculated using Eq. (5), where percentage of life remaining is given by dividing the sum of all $\mathrm{LLP}_{\text {cycles }}$ for a given thrust $\mathrm{T}$ by the sum of all limits for that same thrust. 


$$
L L P_{\text {life }}=\frac{\sum L L P_{T}}{\sum \text { Limits }_{T}}
$$

Once the graph is created to have an overall view of engine maintenance status at each phase-out, the program will allow for changes to be made to the thrust setting, SV schedule or SV workscope definitions. These parameters are changed to try and match the point in time when an engine reaches the phase-out conditions to the engine phaseout dates. After using the graph to attempt to define a maintenance plan that best fits the phase-outs, a decision must be made on which engine will be phased-out at what moment. These choices will be needed to calculate the phaseout costs that will result.

\section{F. Cost Calculation}

To predict the DMC some basic Life Cycle Costing theory is applied. ${ }^{5}$ The approach for the cost analysis is based on five steps: Develop a representative cost structure, Collect data to support the cost structure, Develop cost estimating relationships from the cost data, Formulate the complete cost model, Validate and exercise the model. This section will introduce the equations used for the cost prediction. The equations used in this section are based firstly on the cost data analysis and then confirmed by discussions with a technical purchasing manager assigned to an engine maintenance account.

The final output is the cost of the maintenance plan, which is broken-down into the DMC of each individual engine and the DMC of the engine 'fleet' as a whole. The DMC of an individual engine is measured in cost per hour $(\mathrm{CPH})$ and is given by Eq. (6).

$$
C P H_{D M C}=\left(C_{\text {contract }}+C_{L L P}+C_{P h O}+C_{\mathrm{Re} n t a l}\right) / F H
$$

The first cost factor is the ESPH contract. The monthly rate that must be paid is split into a set cost and the bucket, Eq. (7). X\% goes to the company to which engine maintenance is outsourced and the remaining goes to the bucket. The money in the bucket is kept aside to pay for SVs or give to the engine lessor at the end of the lease contract.

$$
C_{\text {contract }}=\left[R_{T} \times X \% \times\left(F H-F H_{O}\right)\right]+\left[R_{T} \times(1-X \%) \times\left(F H-F H_{O}\right)\right]
$$

The cost brought about by the LLPs is calculated using Eq. (8). Here the value of the stub life remaining is added to the amount of money invested in LLPs to obtain the total cost of the LLP management choices.

$$
C_{L L P}=L L P_{\text {loss }} \times \text { Value }_{L L P}+L L P_{\text {invest }}
$$

The last two cost factors are the phase-out cost and the engine rental cost. The phase-out cost is the financial penalty that must be paid for not meeting phase-out conditions, Eq. (9), these vary depending on the lease contract. The engine rental cost, Eq. (10), is the cost of leasing in additional engines when there are no spare engines available to cover for an engine going to shop.

$$
\begin{gathered}
C_{P h O}=P_{P h O} \\
C_{\text {Rental }}=R_{\text {rent }} \times d a y s
\end{gathered}
$$

Using this cost algorithm the program calculates the cost of an individual engine. The DMC cost of the engine 'fleet' as a whole is given by the summation of the $\mathrm{CPH}_{\mathrm{DMC}}$ of each individual engine, Eq. (11).

$$
D M C_{\text {Total }}=\sum E S N_{i}\left(C P H_{D M C}\right)
$$

\section{G. Scenario Generation}


The output of the tool can be defined as the maintenance plan and its DMC. It is now interesting to generate different scenarios so that a comparison can be made between them and decide on which one to follow. To generate new scenarios the tool uses an iterative algorithm principle. ${ }^{6}$ This will help identify lowest cost management solution considering a wide range of possible combinations of all cost parameters. This allows for the checking of the sensitivity of the DMC to particular variables, which helps identify the factors that have the greatest impact on cost.

The iterative algorithm approach applied to this model consists of five steps, as depicted in figure 9. In the first step an SV schedule is created using the forecasted aircraft AUR. In the second step SVs workscopes are defined. The third step is deciding, using the phase-out overview, which engines to phase-out at which moment. Once the previous three steps have been fully defined the DMC for the maintenance plan is calculated in the fourth step. The final step in the iteration is where reconfiguration of the thrust settings, SV schedule or workscope definitions are considered and, if interesting, introduced. Using this iteration, employees are able to analyze the impact of these three variables on the DMC and create a list of several interesting engine maintenance scenarios trying to optimize either operations or cost. These scenarios are then analysed, and the most interesting ones are presented to management to decide on the most appropriate maintenance plan.

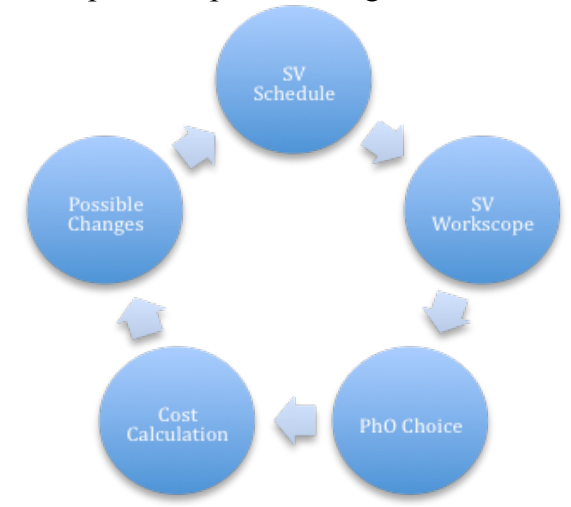

Figure 13. Iterative algorithm principle

\section{H. Control}

Finally the failure-based SVs are seen as unscheduled and not forecasted by the tool. However, these SVs have to be dealt with in some way as they can disrupt maintenance scheduling. In this case these unscheduled SVs will be introduced into the tool when they occur, this will at least show the impact they have on the maintenance plan and help decide the workscope of the SV so that its impact is minimized.

\section{Conclusion}

The engine maintenance-planning model, with the use of the decision support tool, proves to be an effective way to deal with the operational and financial planning obstacles faced by engine management. The tool brings together the forecasting, planning, and controlling aspects of maintenance. It provides users with a clear overview of the engine fleet's maintenance status in future years, it allows for the maintenance plan definition taking into account phase-out moments, and most important it directly shows the impact of unscheduled incidents on the overall plan. This last function is useful in order for management to react to unexpected events and decide on how to modify the original plan in the most cost efficient manner. It helps compare different possibilities before taking decisions that affect both operational and economical aspects related to engine maintenance.

The tool is currently limited to analyzing the impact of changes that can be made by E\&M to factors that influence engine MRO: engine thrust setting, SV schedule, and SV workscopes. These variables directly define the engine maintenance philosophy dealing with engine time on-wing and LLP management, which control the final maintenance cost. However, there is theory that shows that fuel burn should also play an important role in engine MRO decisions. ${ }^{11,12}$ However, for airlines with ESPH contracts this is usually not one of the engine removal reasons that classify an engine for an SV. In any case, the increase in fuel consumption with time is currently being introduced into the tool to allow airlines to decide whether fuel consumption should be negotiated as an engine removal factor.

This model has other interesting aspects besides the mere economical ones. One of the stronger ones is the bringing together of the information from the engineering, purchasing, and lease departments into one planning tool. This improves communication between the departments, forcing them to sit down and agree on a strategy to follow.

\section{Acknowledgments}

The authors would like to thank Transavia Airlines CV for their collaboration in the development of this maintenance-planning model. Special acknowledgement goes out to Jan Maas (Purchasing Manager) and Natascha Martin (System Engineer) for their support throughout the tool definition process, and to Xavi Amoros for cooperating with the programming of the tool. 


\section{References}

${ }^{1}$ Ackert, S, "Engine Maintenance Concepts for Financiers," 2010.

${ }^{2}$ Buyers, T., "737 Next Generation Maintenance Cost Working Group,” TOPICS Conference, Boeing, Berlin, 2006.

${ }^{3}$ Boeing: Fleet Management Slides, 2008

${ }^{4}$ Bowman, R.A, Scmee, J., "Pricing and Managing a Maintenance Contract for a Fleet of Aircraft Engines," Union College Management Institue, New York, 2001.

${ }^{5}$ Ghobbar, A.A., "AE4-418 Life Cycle Costing and Engineering course notes,” Delft, 2007.

${ }^{6}$ Hinow, M., Waldron, M., Müller, L., "Substation Life Cycle Cost Management Supported By Stochastic Optimization Algorithm," 10 .

${ }^{7}$ Hessburg, Jack, Air Carrier MRO Handbook: Maintenance, Repair, and Overhaul, McGraw-Hill, New York, 2001, Chap.

${ }^{8}$ Higgins, Lindley R., Maintenance Engineering Handbook, McGraw-Hill, New York, 1990.

${ }^{9}$ Kinnison, H.A., Aviation Maintenance Management, McGraw-Hill, New York, 2004. Chaps 3, 9, 15.

${ }^{10}$ Langholf, P., "Managing Cold End Cost," Aero-Engines Maintenance \& Overhaul Conference, MTU Aero Engines, Paris, 2008.

${ }^{11}$ Ouwehand, A.M, "Engine Maintenance: Discerning Fact From Fiction,” Msc Thesis, Aerospace Engineering, Technical University Delft, Delft, 2009.

${ }^{12}$ Wong, J.S, Scanlan, J.P, Eres, M.H, "Modeling the Life Cycle Cost of Aero-Engine Maintenance," University of Southampton, Southampton. 